\title{
ARE WEIGHTED GAMES SUFFICIENTLY GOOD FOR BINARY VOTING?
}

\author{
SASCHA KURZ
}

\begin{abstract}
Binary "yes"-"no" decisions in a legislative committee or a shareholder meeting are commonly modeled as a weighted game. However, there are noteworthy exceptions. E.g., the voting rules of the European Council according to the Treaty of Lisbon use a more complicated construction. Here we want to study the question if we loose much from a practical point of view, if we restrict ourselves to weighted games. To this end, we invoke power indices that measure the influence of a member in binary decision committees. More precisely, we compare the achievable power distributions of weighted games with those from a reasonable superset of weighted games.
\end{abstract}

JEL classification: $\mathrm{C} 61, \mathrm{C} 71$

Keywords: power measurement; weighted games

\section{INTRODUCTION}

Consider a family, consisting of mother Ann, father Bob, and the two kids Cathrin and Dave, deciding on their joint weekend activities. In a weighted game each voter $i$ has a non-negative weight $w_{i}$ and a proposal is accepted if the sum of the weights of its supporters meets or exceeds a positive quota $q$. As an abbreviation we write $\left[q ; w_{1}, \ldots, w_{n}\right]$ for a weighted game with $n$ voters. The example $[3 ; 3,2,1,1]$ (where we number in alphabetical order) might model a slightly parents biased, especially mother biased, decision rule. Another voting rule might be that either both parents or both kids have to agree. It can be shown that no representation as a weighted game exists. Since all family members have equal opportunities to influence the final decision, all reasonable measures of voting power assign equal power to all members. The weighted games $[2 ; 1,1,1,1]$ or $[3 ; 1,1,1,1]$ have the same power distribution (while $[3 ; 3,2,1,1]$ has not). If we only care about the resulting power distribution we can also choose a weighted game in our situation. Even more practically, we may accept a weighted game as a plausible replacement of the original voting rule if the corresponding power distribution does not differ too much. Here we want to study the question how large this difference can be in the worst case. 
A related problem is the so-called inverse power index problem, where one wants to determine the game whose power distribution is closest to a predefined target power distribution. For more details see e.g. [De et al.(2017)] and the reference cited therein. [Alon and Edelman(2010)] have shown that some target power distributions, where most players have negligible or even zero power, like e.g. $(0.75,0.25,0, \ldots, 0)$, cannot be approximated too closely by the power distribution of any game. ${ }^{1}$ Our setting differs as follows. Instead of all non-negative vectors summing to one, we only consider the power distributions attained by a superset of weighted games as possible target power distributions and ask to what extend they can be approximated by the power distribution of a weighted game.

\section{Preliminaries}

By $N=\{1, \ldots, n\}$ we denote the set of voters. A simple game is a surjective and monotone mapping $v: 2^{N} \rightarrow\{0,1\}$ from the set of subsets of $N$ into a binary output $\{0,1\}$. Monotone means $v(S) \leq v(T)$ for all $\emptyset \subseteq S \subseteq T \subseteq N$. A simple game $v$ is weighted if there exist weights $w_{1}, \ldots, w_{n} \in \mathbb{R}_{\geq 0}$ and a quota $q \in \mathbb{R}_{>0}$ such that $v(S)=1$ iff $w(S):=\sum_{i \in S} w_{i} \geq q$. As stated in the introduction, we abbreviate a weighted game by $\left[q ; w_{1}, \ldots, w_{n}\right]$, Two voters $i$ and $j$ are called symmetric, in a given simple game $v$, if $v(S \cup\{i\})=v(S \cup\{j\})$ for all $\emptyset \subseteq S \subseteq N \backslash\{i, j\}$. Voter $i \in N$ is a null voter if $v(S)=v(S \cup\{i\})$ for all $\emptyset \subseteq S \subseteq N \backslash\{i\}$. Given two simple games $v$ and $v^{\prime}$ we define their intersection $v \wedge v^{\prime}$ via $\left(v \wedge v^{\prime}\right)(S)=\min \left\{v(S), v^{\prime}(S)\right\}$ for all $S \subseteq N$. Similarly, the union is given by $\left(v \vee v^{\prime}\right)(S)=\max \left\{v(S), v^{\prime}(S)\right\}$ for all $S \subseteq N$. The non-weighted decision rule from the introduction can be written as $[2 ; 2,0,1,1] \wedge[2 ; 0,2,1,1]$ or $[2 ; 1,1,0,0] \vee[2 ; 0,0,1,1]$. It is well known that every simple game can be written as the intersection (or union) of a finite list of weighted games. Also combinations of $\wedge$ and $\vee$ are used in practice. An example is given by the voting system of the European Council according to the Treaty of Lisbon. For $n=27$ (or $n=28$ ) countries the voting system can be written as $v=\left([0.55 n ; 1, \ldots, 1] \wedge\left[0.65 ; p_{1}, \ldots, p_{n}\right]\right) \vee[n-3 ; 1, \ldots, 1]$, where $p_{i}$ denotes the relative population of country $i$. As remarked by [Kirsch and Langner(2011)], dropping the union with $[n-3 ; 1, \ldots, 1]$ has almost no impact on the characteristic function $v$ or corresponding power distributions. Consisting of a Boolean combination, i.e., $\wedge$ 's and $\vee$ 's, of three weighted games the stated representation of the voting system of the European Council (according to the Treaty of Lisbon) is relatively compact. For a general simple game for $n$ voters an exponential number of weighted games

\footnotetext{
${ }^{1}$ More precisely, [Alon and Edelman(2010)] show such a result for the Banzhaf index. Results for other power indices have been obtained by [Kurz(2016)].
} 
can be necessary in the worst case, see [Faliszewski et al.(2009)]. Writting down the characteristic function $v$ explicitly also has exponential complexity, while a weighted game can be written by listing $n$ integer weights and a quota. Framed differently, the number of simple games is many orders of magnitudes larger than the number of weighted games.

As a class of binary voting systems between simple games and weighted games we consider complete simple games, see [Carreras and Freixas(1996)]. They are based on Isbell's desirability relation, see [Isbell(1956)], where we write $i \succeq j$ if $v(S \cup\{i\}) \geq v(S \cup\{j\})$ for all $S \subseteq N \backslash\{i, j\}$ for two voters $i, j \in N$. A simple game $v$ is called complete if this relation is complete, i.e., if for all $i, j \in N$ we have $i \succeq j$ or $j \succeq i$. Two players $i, j \in N$ are symmetric iff $i \succeq j$ or $j \succeq i$. The relation $\succeq$ induces an ordering of the players, which is satisfied in many practical applications. E.g. the voting systems of the European Council (according to the Treaty of Lisbon and also those before) are complete simple games. Here we use the standard assumption $1 \succeq 2 \succeq \cdots \succeq n$ and note that $\operatorname{SSI}(v)$ and $\operatorname{BZI}(v)$ are nonincreasing vectors for every complete simple game $v$. In order to uniquely characterize a complete simple game $v$ we can list all subsets $S \subseteq N$ such that $v(S)=1$ and for every $i \in S, j \notin S$ with $i<j$ we have $v(S \backslash\{i\} \cup\{j\})=0$. For our example $[3 ; 3,2,1,1]$ those subsets are given by $\{1\}$ and $\{2,4\}$. In our example $[2 ; 2,0,1,1] \wedge[2 ; 0,2,1,1]$ the voters 1 and 2 as well as voters 3 and 4 are symmetric. For all other pairs of different voters we neither have $i \succeq j$ nor $j \succeq i$, i.e., the game is not complete.

A power index $p$ is a mapping from the set of simple (or weighted) games on $n$ voters into $\mathbb{R}^{n}$. By $p_{i}(v)$ we denote the $i$ th component of $p(v)$, i.e., the power of voter $i$. Here we consider two of the most commonly used power indices, i.e., the Shapley-Shubik index

$$
\operatorname{SSI}_{i}(v)=\sum_{S \subseteq N \backslash\{i\}} \frac{|S| ! \cdot(n-|S|-1) !}{n !} \cdot(v(S \cup\{i\})-v(S))
$$

and the Penrose-Banzhaf index

$$
\operatorname{BZI}_{i}(v)=\frac{\sum_{S \subseteq N \backslash\{i\}}(v(S \cup\{i\})-v(S))}{\sum_{j \in N} \sum_{S \subseteq N \backslash\{j\}}(v(S \cup\{j\})-v(S))} .
$$

For our first example $v=[3 ; 3,2,1,1]$ we have $\operatorname{SSI}(v)=\frac{1}{12} \cdot(7,3,1,1) \approx$ $(0.5833,0.25,0.0833,0.0833)$ and $\operatorname{BZI}(v)=\frac{1}{10} \cdot(5,3,1,1)=(0.5,0.3,0.1,0.1)$. As a measure for the distance between two different power distributions $x, y \in \mathbb{R}^{i}$ we use the Manhattan distance $d_{1}(x, y)=\sum_{i=1}^{n}\left|x_{i}-y_{i}\right|$ and the Chebyshev distance $d_{\infty}(x, y)=\max _{1 \leq i \leq n}\left|x_{i}-y_{i}\right|$. For the above two power distributions the Manhattan distance is $\frac{1}{6} \approx 0.1667$ and the Chebyshev distance is $\frac{1}{12} \approx 0.0833$. 


\section{Results}

In the introduction we have noticed that $[2 ; 1,1,1,1]$ as well as $[3 ; 1,1,1,1]$ yield the power distribution $(0.25,0.25,0.25,0.25)$ both for the ShapleyShubik and the Banzhaf index. In Table 1 we state the number of different power distributions for the Shapley-Shubik and the Banzhaf index that are attained by weighted games with $n \leq 8$ voters. The corresponding numbers for complete simple games are listed in Table 2.

\begin{tabular}{lrrrrrr}
\hline$n$ & 3 & 4 & 5 & 6 & 7 & 8 \\
\hline \# SSI & 4 & 11 & 53 & 536 & 14188 & 1364907 \\
\# BZI & 4 & 12 & 57 & 555 & 14720 & 1366032
\end{tabular}

TABLE 1. Number of different vectors $\operatorname{SSI}(v)$ and BZI $(v)$ for weighted games $v$ with $n$ voters.

\begin{tabular}{lrrrrrr}
\hline$n$ & 3 & 4 & 5 & 6 & 7 & 8 \\
\hline \# SSI & 4 & 11 & 53 & 536 & 17973 & 6314952 \\
\# BZI & 4 & 12 & 57 & 555 & 18600 & 4616157
\end{tabular}

TABLE 2. Number of different vectors $\operatorname{SSI}(v)$ and BZI $(v)$ for complete simple games $v$ with $n$ voters.

We observe that the counts coincide for $n \leq 6$, which is no surprise for $n \leq 5$, since every complete simple game consisting of at most 5 voters is weighted. However, for $n=6$ voters there exist $1171-1111=60$ complete simple games that are not weighted. Nevertheless, the power distributions according to the Shapley-Shubik index or the Banzhaf index of these 60 nonweighted complete simple games are also exactly attained by weighted games, respectively. So, if we are only interested in the resulting power distribution, then including complete non-weighted games comes with no benefit for $n \leq 6$ voters. For $n \in\{7,8\}$ we do not have such a strong result. Here the number of attained power distributions for complete simple games is significantly larger. This goes in line with the fact that there are $4313-29373=14940$ and $16175188-2730164=13445024$ non-weighted complete simple games for $n=7$ and $n=8$ voters, respectively. There we can only give a worstcase bound for the minimum distance between the power distribution of a complete simple game and a weighted game. To this end, we denote the set of weighted games with $n$ voters by $\mathcal{W G}(n)$ and the set of complete simple games with $n$ voters by $\mathcal{C G}(n)$. Moreover, let

$$
\omega_{a}^{p}(n):=\max \left\{\min \left\{d_{a}(p(c), p(v)): v \in \mathcal{W G}(n)\right\}: c \in \mathcal{C} \mathcal{G}(n)\right\},
$$


where $a \in\{1, \infty\}$ and $p \in\{\mathrm{SSI}, \mathrm{BZI}\}$, be the worst-case distance between the power distribution $p(c)$ of a complete simple game $c$ and the power distribution $p(v)$ of its best approximation by a weighted game $v$.

\section{Proposition 1.}

$$
\begin{array}{ll}
\omega_{1}^{\mathrm{SSI}}(7)=0.0666667 & \omega_{1}^{\mathrm{SSI}}(8)=0.0666667 \\
\omega_{\infty}^{\mathrm{SSI}}(7)=0.0166667 & \omega_{\infty}^{\mathrm{SSI}}(8)=0.0154762 \\
\omega_{1}^{\mathrm{BZI}}(7)=0.0599700 & \omega_{1}^{\mathrm{BZI}}(8)=0.0567084 \\
\omega_{\infty}^{\mathrm{BZI}}(7)=0.0173913 & \omega_{\infty}^{\mathrm{BZI}}(8)=0.0139124
\end{array}
$$

Proof. The proof is obtained by a computer enumeration. First, we loop over all elements $v$ in $\mathcal{W G}(n)$ and store the corresponding power distributions $p(v)$ in a $k$-d-tree (a data structure for storing multi-dimensional geometrical data). Afterwords, we loop over all elements $c$ in $\mathcal{C G}(n)$, compute $p(c)$, and perform a nearest neighbor search within the previously computed $k$-d-tree. Let $v$ denote the nearest neighbor that minimizes $d_{a}^{p}(p(v), p(c))$. Eventually update the worst-case distance with $d_{a}^{p}(p(v), p(c))$.

As an example we state that the complete simple game attaining $\omega_{\infty}^{\mathrm{BZI}}(7)=$ 0.0173913 is uniquely characterized by the subsets $\{3,4,5,6,7\},\{2,3,5,6\}$, and $\{1,3,7\}$. For $n=8$ the extremal complete simple games all contain a unique null voter. We remark that the same enumeration is computationally infeasible for $n=9$ voters since the numbers $\# \mathcal{W G}(9)=993061482$ and $\# \mathcal{C G}(9)=284432730174$ are quite large. (See e.g. [Kartak et al.(2015)] and [Kurz and Tautenhahn(2013)] for the details.) So, for $n \geq 9$ we can only state lower bounds for $\omega_{a}^{p}(n)$ :

\section{Proposition 2.}

$$
\begin{array}{cll}
\omega_{1}^{\mathrm{SSI}}(9) \geq 0.0634922 & \omega_{1}^{\mathrm{SSI}}(10) \geq 0.0634922 & \omega_{1}^{\mathrm{SSI}}(11) \geq 0.0591627 \\
\omega_{\infty}^{\mathrm{SSI}}(9) \geq 0.0130953 & \omega_{\infty}^{\mathrm{SSI}}(10) \geq 0.0123016 & \omega_{\infty}^{\mathrm{SSI}}(11) \geq 0.0109308 \\
\omega_{1}^{\mathrm{BZI}}(9) \geq 0.0562 & \omega_{1}^{\mathrm{BZI}}(10) \geq 0.0552 & \omega_{1}^{\mathrm{BZI}}(11) \geq 0.0552 \\
\omega_{\infty}^{\mathrm{BZI}}(9) \geq 0.0110 & \omega_{\infty}^{\mathrm{BZI}}(10) \geq 0.0106 & \omega_{\infty}^{\mathrm{BZI}}(11) \geq 0.0100
\end{array}
$$

Proof. Let $a \in\{1, \infty\}$ and $p \in\{$ SSI, BZI $\}$. In $[\operatorname{Kurz}(2012)]$ the inverse power index problem for the Shapley-Shubik index with respect to the Manhattan distance $d_{1}(\cdot, \cdot)$ and the Chebyshev distance $d_{\infty}(\cdot, \cdot)$ within the class of weighted, complete simple, or simple games was formulated as an integer linear programming (ILP) problem, which can be solved exactly even for $n>9$, where the number of weighted games is unknown. For the Banzhaf 
index the problem whether a solution of the inverse power index problem with distance at most $\delta$ exists can be formulated as an ILP. Using the bisection method for $\delta$ the problem can be solved exactly by a sequence of ILPs, see [Kurz and Napel(2014), Appendix A] for the details. Thus, given a complete simple game $c$ with $n$ voters we can compute the corresponding power distribution $p(c)$ and exactly solve the inverse power index problem within $\mathcal{W G}(n)$. If $v$ is a weighted game that minimizes $d_{a}(p(c), p(v))$, then $d_{a}(p(c), p(v))$ is a lower bound for $\omega_{a}^{p}(n)$. As heuristic candidates for the complete simple game $c$ we have used the extremal ones of Proposition 1 and added a suitable number of null voters.

We remark that we have also tried to use some randomly chosen complete simple games for $c$ in Proposition 2. However, the resulting lower bounds for $\omega_{a}^{p}(n)$ are rather small. As an example, the value $\omega_{1}^{\mathrm{SSI}}(7)=0.0666667$ is attained by the complete simple game $c$ characterized by the subsets $\{4,5,6,7\},\{2,4\}$, and $\{1\}$. If we add a null voter, the Shapley-Shubik index is given by $(0.5024,0.1857,0.1024,0.1024,0.03571,0.03571,0.03571,0)$ with best possible approximation $[84 ; 38,27,19,16,9,9,3,0]$, which also shows $\omega_{1}^{\mathrm{SSI}}(8) \geq 0.0666667$.

For the voting system $c$ of the European Council according to the Lisbon Treaty we cannot solve the inverse power index problem exactly. However, for all $a \in\{1, \infty\}$ and all $p \in\{\mathrm{SSI}, \mathrm{BZI}\}$ we can find a weighted game $v$ with $d_{a}(p(c), p(v))<10^{-5}$, which goes in line with the computational experiments in [Kurz and Napel(2014)].

\section{Conclusion}

Does it pay off to use complete simple games instead of weighted games as binary voting systems? If only the resulting power distributions for the Shapley-Shubik or the Banzhaf index are relevant, then the answer is probably no. Whether the worst-case deviations stated in Proposition 1 can be regarded as negligible might depend on the application. For $n>8$ voters our computational experiments suggest that the worst-case deviations might even go down with an increasing number of voters. Proving this claim rigorously might be a hard technical challenge.

We have chosen complete simple games as a reasonable superset of weighted games since the underlying ordering of the players can be assumed in many applications. Another reason is that the class of simple games is really large $^{2}$ and realizes a lot of power distributions. E.g., the parameterized target power distribution $\beta(n)=\frac{1}{2 n-1} \cdot(2, \ldots, 2,1) \in \mathbb{R}^{n}$ has been studied by

\footnotetext{
${ }^{2}$ There are at least $2^{\left(\sqrt{\frac{2}{3} \pi} \cdot 2^{n}\right) /(n \sqrt{n})}$ complete simple games, see [Peled and Simeone(1985)], less than $2^{2^{n}}$ simple games, and at most $2^{n^{2}-n+1}$ weighted games, see [Zunic(2004)].
} 
[Kurz and Napel(2014)]. For $6 \leq n \leq 18$ there exists a simple game $v_{n}$ such that $\operatorname{SSI}\left(v_{n}\right)=\beta(n)$, while the best approximation within $\mathcal{W G}(n)$ seems to have a deviation of order $\Theta\left(\frac{1}{n}\right)$. At the very least our values for $\omega_{a}^{p}(n)$ give a lower bound for the corresponding situation where we enlarge the possible target power distributions to those of simple games.

\section{REFERENCES}

[Alon and Edelman(2010)] Alon, N., Edelman, P. H., 2010. The inverse Banzhaf problem. Social Choice and Welfare 34 (3), 371-377.

[Carreras and Freixas(1996)] Carreras, F., Freixas, J., 1996. Complete simple games. Mathematical Social Sciences 32 (2), 139-155.

[De et al.(2017)] De, A., Diakonikolas, I., Servedio, R. A., 2017. The inverse Shapley value problem. Games and Economic Behavior 105, 122-147.

[Faliszewski et al.(2009)] Faliszewski, P., Elkind, E., Wooldridge, M., 2009. Boolean combinations of weighted voting games. In: Proceedings of The 8th International Conference on Autonomous Agents and Multiagent Systems-Volume 1. International Foundation for Autonomous Agents and Multiagent Systems, pp. 185-192.

[Isbell(1956)] Isbell, J. R., 1956. A class of majority games. The Quarterly Journal of Mathematics 7 (1), 183-187.

[Kartak et al.(2015)] Kartak, V. M., Ripatti, A. V., Scheithauer, G., Kurz, S., 2015. Minimal proper non-IRUP instances of the one-dimensional cutting stock problem. Discrete Applied Mathematics 187, 120-129.

[Kirsch and Langner(2011)] Kirsch, W., Langner, J., 2011. Invariably suboptimal: An attempt to improve the voting rules of the Treaties of Nice and Lisbon. Journal of Common Market Studies 49 (6), 1317-1338.

[Kurz(2012)] Kurz, S., 2012. On the inverse power index problem. Optimization 61 (8), 989-1011.

[Kurz(2016)] Kurz, S., 2016. The inverse problem for power distributions in committees. Social Choice and Welfare 47 (1), 65-88.

[Kurz and Napel(2014)] Kurz, S., Napel, S., 2014. Heuristic and exact solutions to the inverse power index problem for small voting bodies. Annals of Operations Research $215(1), 137-163$.

[Kurz and Tautenhahn(2013)] Kurz, S., Tautenhahn, N., 2013. On Dedekind's problem for complete simple games. International Journal of Game Theory 42 (2), 411-437.

[Peled and Simeone(1985)] Peled, U. N., Simeone, B., 1985. Polynomial-time algorithms for regular set-covering and threshold synth esis. Discrete Applied Mathematics $12(1), 57-69$.

[Zunic(2004)] Zunic, J., 2004. On encoding and enumerating threshold functions. IEEE Transactions on Neural Networks 15 (2), 261-267.

Sascha Kurz, Department of Mathematics, University of Bayreuth, Germany. Tel: +49921 557353. E-mall: Sascha.kurz@uni-bayreuth.de 